\title{
The Characteristics of Cognitive Impairment and Their Effects on Functional Outcome After Inpatient Rehabilitation in Subacute Stroke Patients
}

\author{
Soo Ho Park, MD, Min Kyun Sohn, MD, Sungju Jee, MD, Shin Seung Yang, MD \\ Department of Rehabilitation Medicine, Chungnam National University School of Medicine and \\ Regional Cardiocerebrovascular Center, Chungnam National University Hospital, Daejeon, Korea
}

\begin{abstract}
Objective To determine the frequency and characteristics of vascular cognitive impairment (VCI) in patients with subacute stroke who underwent inpatient rehabilitation and to analyze whether cognitive function can predict functional assessments after rehabilitation.

Methods We retrospectively reviewed the medical records of patients who were admitted to our rehabilitation center after experiencing a stroke between October 2014 and September 2015. We analyzed the data from 104 patients who completed neuropsychological assessments within 3 months after onset of a stroke.

Results Cognitive impairment was present in 86 out of 104 patients (82.6\%). The most common impairment was in visuospatial function $(65,62.5 \%)$ followed by executive function $(63,60.5 \%)$, memory $(62,59.6 \%)$, and language function $(34,32.6 \%)$. Patients with impairment in the visuospatial and executive domains had poor scores of functional assessments at both admission and discharge $(\mathrm{p}<0.05)$. A multivariate analysis revealed that age $(\beta=$ $-0.173)$ and the scores on the modified Rankin Scale $(\beta=-0.178)$, Korean version of the Modified Barthel Index $(\mathrm{K}-\mathrm{MBI})(\beta=0.489)$ at admission, and Trail-Making Test A (TMT-A) $(\beta=0.228)$ were related to the final K-MBI score at discharge (adjusted $\mathrm{R}^{2}=0.646$ ).

Conclusion In our study, VCI was highly prevalent in patients with stroke. TMT-A scores were highly predictive of their final K-MBI score. Collectively, our results suggest that post-stroke executive dysfunction is a significant and independent predictor of functional outcome.
\end{abstract}

Keywords Stroke, Cognitive symptom, Outcome assessment

Received October 13, 2016; Accepted March 28, 2017

Corresponding author: Min Kyun Sohn

Department of Rehabilitation Medicine and Regional Cardiocerebrovascular Center, Chungnam National University Hospital, 282 Munhwa-ro, Junggu, Daejeon 35015, Korea. Tel: +82-42-338-2416, Fax: +82-42-256-6056, E-mail: mksohn@cnu.ac.kr

ORCID: Soo Ho Park (http://orcid.org/0000-0002-1815-5196); Min Kyun Sohn (http://orcid.org/0000-0002-2548-545X); Sungju Jee (http://orcid. org/0000-0002-9400-9609); Shin Seung Yang (http://orcid.org/0000-0002-6831-1556).

(c) This is an open-access article distributed under the terms of the Creative Commons Attribution Non-Commercial License (http://creativecommons.org/ licenses/by-nc/4.0) which permits unrestricted noncommercial use, distribution, and reproduction in any medium, provided the original work is properly cited. Copyright ( 2017 by Korean Academy of Rehabilitation Medicine 


\section{INTRODUCTION}

One in three individuals will experience a stroke, dementia, or both during their lifetime [1]. Moreover, up to $64 \%$ of people who have experienced a stroke have some degree of cognitive impairment [2]. Cognitive impairment that is caused by or associated with vascular factors is referred to as vascular cognitive impairment (VCI) [3]. VCI encompasses a variety of cognitive deficits that range from relatively mild VCI with no dementia to more severe vascular dementia. Simply, VCI is an impairment related to a clinical stroke or subclinical vascular brain injury that affects at least one cognitive domain [4]. Because some vascular risk factors are treatable, it may be possible to prevent, postpone, or mitigate VCI.

Several studies have elucidated the frequency and characteristics of cognitive dysfunction in patients who have experienced a stroke $[5,6]$. However, given the lack of satisfactory diagnostic criteria for VCI, different incidence rates have been reported. The particular criteria used by a clinician or investigator to diagnose VCI may greatly affect the epidemiology and treatment strategies. According to the Diagnostic and Statistical Manual of Mental Disorders, fourth edition (DSM-IV) and National Institute of Neurological Disorders and Stroke-Association Internationale pour la Recherche etl'Enseignement en Neurosciences, a diagnosis of vascular dementia requires the presence of memory impairment $[7,8]$. However, patients with vascular dementia often show severe deficits in executive, language, and visuospatial functioning but relatively preserved delayed recall upon formal examination $[7,9,10]$. In addition, the process of identifying memory loss in order to obtain a diagnosis of VCI may slow the identification of deficits in other cognitive domains. Therefore, neuropsychological protocols for evaluating VCI should be sensitive to the disabilities of various cognitive domains.

To help standardize the evaluations of VCI, in 2006, the National Institute of Neurological Disorders and Stroke and Canadian Stroke Network proposed the Vascular Cognitive Impairment Harmonization Standards (VCIHS), which serve as a step towards ensuring that the evaluations of cognitive dysfunction in patients with potential VCI are consistent across multicenter studies [7]. The VCIHS consist of four cognitive domains (i.e., memory, executive/activation, visuospatial, and language).
The Korean version of the VCIHS (K-VCIHS) neuropsychological protocol was adapted from the 60-minute neuropsychology VCIHS protocol. The tests and scales that compose the K-VCIHS protocol have been validated and standardized for Korean subjects [8].

Cognitive dysfunction is known to influence patients' rehabilitation assessments and can be used to predict their functional independence and quality of life after a stroke [11], especially since cognitive functioning has the potential to reduce the efficacy of any effects that training produces. Cognitive impairments are associated with arm motor recovery and are thought to be important factors for selecting the appropriate motor rehabilitation intervention after a stroke [12]. Nys et al. [13] reported that within the first 3 weeks, the most important predictors of long-term functional outcome in first-time stroke patients were neglect and memory. In contrast, Wagle et al. [14] reported that executive function and visuospatial ability were predictors of functional outcome. Given these inconsistent results, it is currently unclear which cognitive domains are related to a patient's functional assessments after an acute stroke.

The purpose of the present study was to determine the frequency and characteristics of VCI in patients with subacute stroke who were admitted to the hospital for rehabilitation. Furthermore, we examined the predictive value of the various cognitive domains in terms of inpatient stroke rehabilitation assessments.

\section{MATERIALS AND METHODS}

\section{Participants}

We performed a retrospective analysis of hospitalized patients after a stroke. Patients were consecutively recruited from our stroke rehabilitation center between October 2014 and September 2015. The inclusion criteria were as follows: hospitalized within 7 days of symptom onset at the neurology or neurosurgery department; confirmed with a stroke by computed tomography or magnetic resonance images; transferred to the rehabilitation center; and completed the 60-minute K-VCIHS neuropsychological assessments within 3 months after stroke onset. Patients who were transferred to other departments for concomitant events or patients who died during rehabilitation were excluded from the analyses. The exact number of patients for study is shown in Fig. 1. 


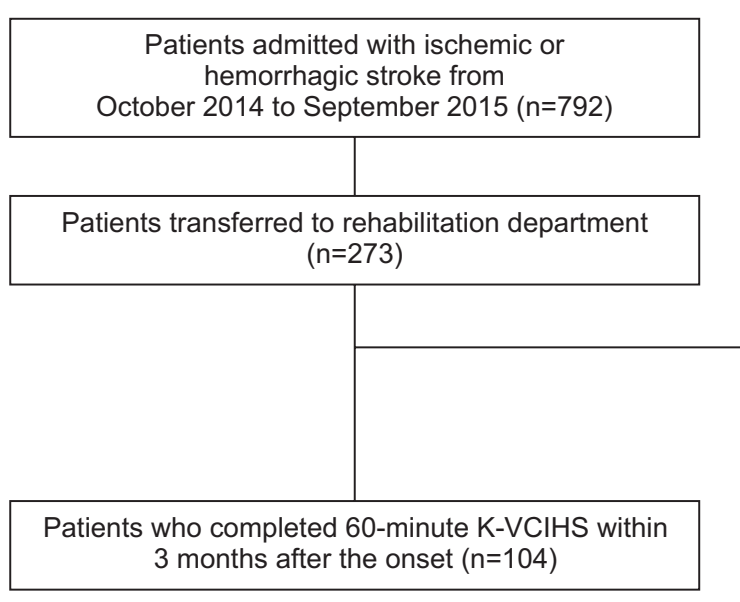

A total of 104 patients met the inclusion criteria. The demographics and clinical characteristics of the patients were obtained by reviewing their electronic medical records. The clinical characteristics of this study's subjects are presented. The mean time of onset to examination was $20.2 \pm 16.1$ days. The mean length of stay at the rehabilitation department was $37.0 \pm 22.7$ days (Table 1).

The Korean versions of the National Institutes of Health Stroke Scale (K-NIHSS), Mini-Mental State Examination (K-MMSE), Modified Barthel Index (K-MBI), and modified Rankin Scale (mRS) were adopted as part of the routine evaluation of patients admitted to the rehabilitation department. Patients were evaluated by an experienced physician at both admission and discharge.

\section{Cognitive evaluation}

The K-VCIHS consist of four cognitive domains and 10 cognitive tests: memory (immediate recall, delayed recall, and recognition tests from the Seoul Verbal Learning Test), visuospatial (Rey Complex Figure Test [RCFT]), language (Korean-Boston Naming Test), and executive function (animal naming test, phonemic fluency test, digit symbol-coding test, Korean Trail-Making Tests A and B [TMT-A, TMT-B]). A trained clinical psychometrician administered the series of tests. A score on each cognitive test was transformed into a standardized z-score:

$\mathrm{z}$-score=[individual score-mean score of the sample]/ standard deviation of the sample.

The z-score was corrected for age and education by using normative corrections for each individual domain. Patients were considered to have cognitive impairments
Table 1. Demographic characteristics of patients $(n=104)$

\begin{tabular}{|lc|}
\hline \multicolumn{1}{|c}{ Characteristic } & Value \\
\hline Age (yr) & $66.5 \pm 11.7$ \\
\hline Sex & \\
\hline Male & 76 \\
\hline Female & 28 \\
\hline Type of paralysis & \\
\hline Right hemiplegia & 54 \\
\hline Left hemiplegia & 44 \\
\hline Double hemiplegia & 6 \\
\hline Type of stroke & \\
\hline Ischemic & 82 \\
\hline Hemorrhagic & 22 \\
\hline Years of education & $9.7 \pm 4.8$ \\
\hline Onset to transfer (day) & $11.6 \pm 10.2$ \\
\hline Onset to examination (day) & $20.2 \pm 16.1$ \\
\hline Length of stay (day) & $37.0 \pm 22.7$ \\
\hline
\end{tabular}

Values are presented as mean \pm standard deviation or number of cases.

in the memory, visuospatial, and language domains if their score was at least two standard deviations (SDs) below the mean [15] on each domain-specific test. Cognitive impairment in the frontal executive domain was defined as a score that was more than two SDs below the mean in two or more of the five domain-specific tests [16]. VCI was defined as impairment in at least one of the four cognitive domains.

\section{Statistical analysis}

We compared the demographics, stroke features, and clinical characteristics of patients after experiencing a 
stroke based upon the presence of cognitive impairment in each domain using a Mann-Whitney test for continuous variables and chi-squared tests for categorical variables. We also compared the z-scores of each test using an analysis of variance. To investigate the variables that might predict the final K-MBI score, which represented the inpatient rehabilitation assessments after a stroke, single linear and multiple regression analyses were applied. All statistically significant variables in the single linear regression analysis were included in the multivariate analysis. A p-value of less than 0.05 was considered statistically significant. All analyses were performed with the Statistical Package for the Social Sciences (SPSS) software program ver. 19.0 (IBM, Armonk, New York, USA).

\section{RESULTS}

\section{Frequency and characteristics of $\mathrm{VCl}$}

Cognitive impairments were present in 86 of the 104 patients (82.6\%). Among the patients with VCI, the most

Table 2. Number of cases with vascular cognitive impairment in each domain

\begin{tabular}{lc}
\hline \multicolumn{1}{c}{ Cognitive domain } & No. of cases (\%) \\
\hline Memory & $62(59.6)$ \\
\hline Visuospatial & $65(62.5)$ \\
\hline Language & $34(32.6)$ \\
Executive & $63(60.5)$ \\
\hline Vascular cognitive impairment & $86(82.6)$ \\
\hline
\end{tabular}

common impairments were noted in the visuospatial function $(65,62.5 \%)$, followed by the executive function $(63,60.5 \%)$, memory function $(62,59.6 \%)$, and language function $(34,32.6 \%)$ (Table 2). The results of the neuropsychological tests and the mean (SD) z-scores for each cognitive domain are shown in Table 3. Compared to the z-scores of the other tests, the z-scores of the RCFT, TMT$A$, and TMT-B were significantly lower $(\mathrm{p}<0.05$, analysis of variance).

\section{Comparisons of the demographic characteristics according to the presence/absence of $\mathrm{VCl}$}

No significant differences in the baseline measures for demographic and stroke characteristics, including age, sex, lesion side, and stroke type, were noted between patients with and without VCI. However, significantly fewer men $(p=0.022)$ and cases of ischemic stroke $(p=0.003)$ were noted among patients with impairment in the language domain. Patients with visuospatial impairment, executive impairment, and VCI had significantly longer durations of rehabilitation hospitalization $(p=0.005$, $\mathrm{p}=0.018$, and $\mathrm{p}=0.029$, respectively) (Table 4 ).

\section{Effects of cognition impairments on functional outcome}

Patients with impairment in the visuospatial and executive domains showed significantly poorer assessments based on their K-NIHSS, K-MMSE, and K-MBI scores at admission and discharge $(\mathrm{p}<0.05)$. Patients with memory impairments exhibited significantly lower admission and

Table 3. Results of neuropsychological tests

\begin{tabular}{|c|c|c|}
\hline Cognitive domain & Neuropsychological tests & Z-scores \\
\hline \multirow[t]{4}{*}{ Memory } & Seoul Verbal Learning Test & \\
\hline & Immediate recall & $-1.86 \pm 1.34$ \\
\hline & Delayed recall & $-1.61 \pm 1.16$ \\
\hline & Recognition & $-1.91 \pm 2.25$ \\
\hline Visuospatial & Rey Complex Figure Test & $-4.32 \pm 4.35^{*}$ \\
\hline Language & Korean-Boston Naming Test-short form & $-1.57 \pm 2.22$ \\
\hline \multirow[t]{5}{*}{ Executive } & Animal Naming Test & $-1.73 \pm 1.34$ \\
\hline & Phonemic Fluency Test & $-1.65 \pm 1.02$ \\
\hline & Korean Trail-Making Test A & $-6.31 \pm 9.75^{*}$ \\
\hline & Korean Trail-Making Test B & $-4.36 \pm 4.64^{*}$ \\
\hline & Digit Symbol Coding Test & $-1.95 \pm 1.17$ \\
\hline
\end{tabular}

Values are presented as mean \pm standard deviation.

${ }^{*} \mathrm{p}<0.05$ analyzed by analysis of variance. 

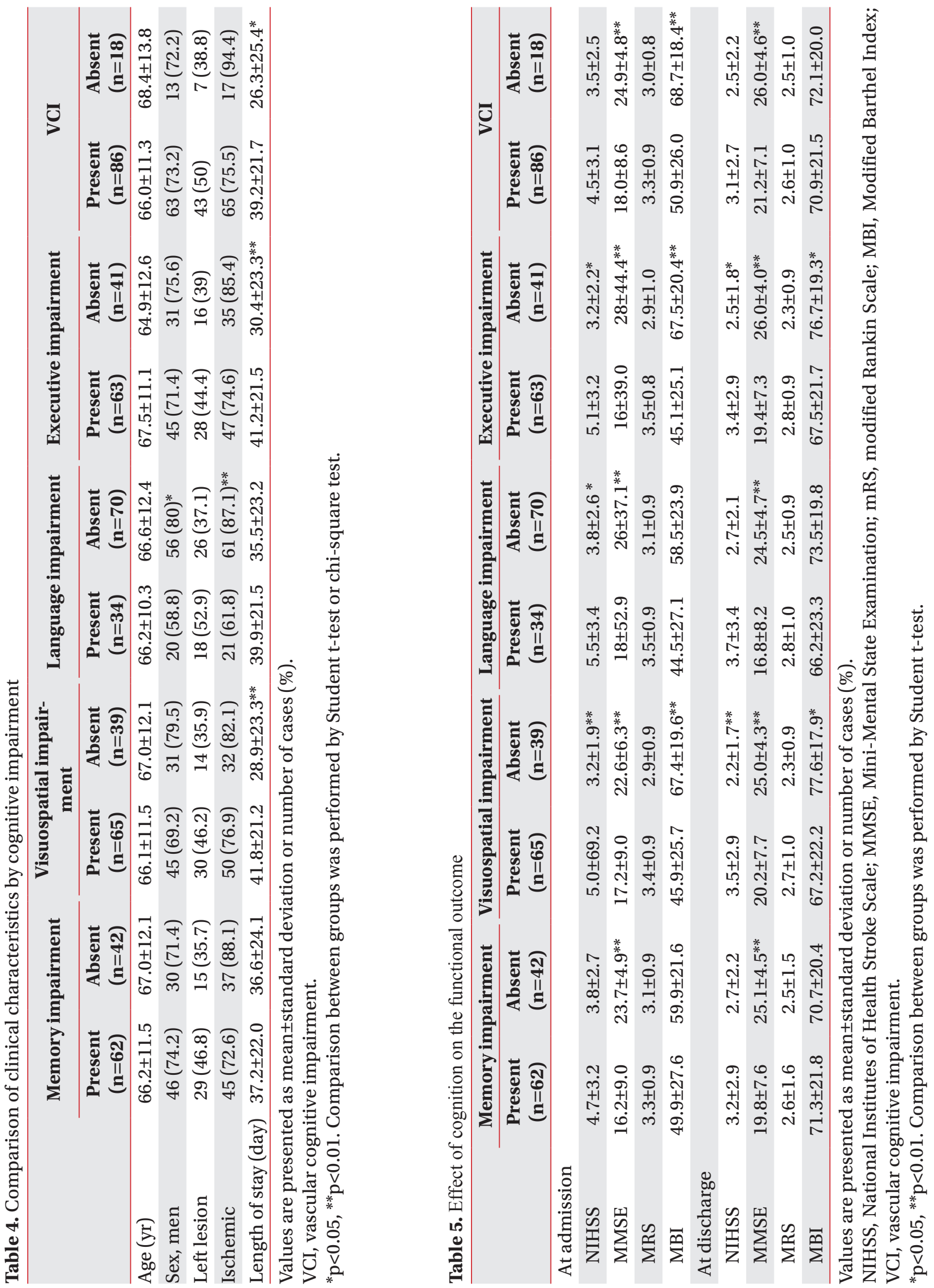
discharge K-MMSE scores $(\mathrm{p}<0.01)$. In addition, patients with impairment in the language domain demonstrated significantly poorer assessments according to their admission K-NIHSS and K-MMSE scores and discharge KMMSE scores $(\mathrm{p}<0.01)$. The K-MMSE scores showed significant differences according to the type of impairment among all cognitive domains at admission and discharge $(p<0.001)$. However, the mRS scores were not significantly different according to the type of impairment among all cognitive domains (Table 5).

\section{Predictors of the final K-MBI scores}

Regression analyses were used to predict the final KMBI scores at discharge. The independent variables were the patients' K-VCIHS data, age, gender, stroke lesion side, stroke type, duration to cognitive evaluation, and the initial mRS, K-MMSE, K-NIHSS, and K-MBI scores. In the single linear regression, the statistically significant variables were age, length of rehabilitation hospitalization (LOS), and the scores for the mRS, K-NIHSS, KMMSE, K-MBI, RCFT, animal naming test, TMT-A, TMT$\mathrm{B}$, and digit symbol-coding test at admission. As such, these variables were included in the multiple regression analysis. The age $(\beta=-0.173), \mathrm{mRS}$ score at admission $(\beta=-$ $0.178), \mathrm{K}-\mathrm{MBI}$ score at admission $(\beta=0.489)$, and TMT-

Table 6. Demographic and cognitive predictors of final MBI analyzed by multiple regression analysis $(n=104)$

\begin{tabular}{lcc}
\hline & Final MBI $(\beta)$ & p-value \\
\hline Age & $-0.173^{*}$ & 0.013 \\
Length of stay & 0.118 & 0.112 \\
\hline MRS & $-0.178^{*}$ & 0.044 \\
NIHSS & -0.156 & 0.066 \\
\hline MMSE & -0.098 & 0.262 \\
MBI & $0.489^{*}$ & 0.000 \\
\hline RCFT & 0.115 & 0.271 \\
\hline Animal Naming & -0.064 & 0.482 \\
\hline TMT-A & $0.288^{*}$ & 0.008 \\
\hline TMT-B & -0.186 & 0.071 \\
\hline Digit Symbol Coding Test & -0.055 & 0.508 \\
\hline
\end{tabular}

mRS, modified Rankin Scale; NIHSS, National Institutes of Health Stroke Scale; MMSE, Mini-Mental State Examination; MBI, Modified Barthel Index; RCFT, Rey Complex Figure Test; TMT-A, Korean Trail-Making Test A; TMT-B, Korean Trail-Making Test B; $\beta$, regression coefficient. Significant data are marked with the asterisk (*).
A score $(\beta=0.228)$ were related to their final K-MBI score (Table 6). The explained variance of the model was 0.646 (adjusted $\mathrm{R}^{2}=0.646$ ).

\section{DISCUSSION}

The aims of the present study were to evaluate the frequency and characteristics of VCI in patients who experienced a subacute stroke and underwent inpatient rehabilitation and to analyze the predictive value of various cognitive domains with regard to the stroke rehabilitation assessments. In this study, a high frequency of VCI was noted within the first 3 months after stroke $(82.6 \%$ of post-stroke survivors). In a previous multicenter study in Korea, the frequency of VCI was $62.6 \%(221 / 353)$ at 3 months [8]. Another study performed in China found that when post-stroke cognitive impairments were defined using the Montreal Cognitive Assessment, MMSE, and Hachinski Ischemia Scale, the prevalence of post-stroke cognitive impairment was $\mathbf{8 0 . 9 7 \% ~ [ 1 7 ] . ~ T h e ~ d i s p a r i t y ~ i n ~}$ VCI frequency among these studies might be related to the differences in study methods including the use of different assessment scales and cutoff values for cognitive impairment. In our study, the subjects were patients who experienced a stroke and were admitted to the hospital for rehabilitation. Therefore, our patients may have exhibited more functional and cognitive deficits. Although specific and standardized diagnostic criteria for VCI currently remain undefined, a substantial prevalence of VCI was found among the patients in our study. For this reason, clinicians should pay careful attention to patients who have a stroke, especially those who experience cognitive problems during the subacute phase, to ensure that cognitive impairments are detected early. Early detection may then help to effectively treat VCI in these patients.

In our study, no significant differences were noted between laterality in the visuospatial and language domain. In the RCFT which assessed visuospatial impairment, patients with lesions in the left hemisphere tended to make more simplifications in their copies than do patients with right-sided lesions. Simplification in patients with left hemisphere damage may be a defect of execution as opposed to one of perception or cognition. Furthermore, simplification errors made by patients with left hemisphere damage may be the product of the left hand's deficient control over fine movement. The Korean-Boston 
Naming Test consists of 60 large ink drawings of items ranging in familiarity. In patients with right frontal damage, some of the drawings elicit responses reflecting perceptual fragmentation. Considering the above explanation, we think that various cognitive domains are not completely independent of each other in the K-VCIHS.

Our results showed that of the cognitive functions examined, the most common impairments were in visuospatial function followed by executive function. Moreover, the patients' $\mathrm{z}$-scores on the subtests for these two cognitive domains (RCFT, TMT-A, and TMT-B) were significantly lower than the scores for the other domainspecific tests. Previous studies have shown that visuospatial and executive functions are especially vulnerable to vascular insults, including those induced by a stroke, since vast territories of the parietal and prefrontal regions are often affected [18-20]. In the present study, visuospatial and executive function impairments were also significantly associated with poor functional assessments at admission and discharge. Completing the subtests of visuospatial and executive function requires motor dexterity (e.g., holding and moving a pen) and speed. Furthermore, because the K-MBI is primarily a measure of the basic activities of daily living and physical dependency, visuospatial and executive function impairments would affect patients' activities of daily living more than would impairments in other cognitive domains.

Patients with executive function impairment showed significantly poorer functional assessments at admission and discharge in this study. Executive function is reportedly a main contributing factor to a patient's rehabilitation participation and overall functional status after rehabilitation [21]. One explanation is related to the poorer functional prognoses in patients with executive dysfunction. For instance, individuals with executive function impairments have difficulty initiating activities, maintaining response consistency, inhibiting impulsive behaviors, and generalizing instructions to other tasks; hence, they may have difficulty internalizing and applying rehabilitation instructions.

An early accurate prediction of a patient's assessments after a stroke is essential for establishing rehabilitation goals. Many studies have attempted to predict patient outcomes using a variety of demographic, radiological, neurophysiological, and neurological variables [22]. A limitation of these studies is that the findings are based on simple associations between the predictor variable and the outcome measure. However, it is important to determine whether a given factor remains an independent predictor for the desired outcome. Here, the TMTA in the K-VCIHS was a cognitive predictor of the final $\mathrm{K}-\mathrm{MBI}$ score at discharge. This indicates that in the present study, the TMT-A served as a better predictor of the functional state of stroke patients after rehabilitation than did the other tests. One possible explanation for this relationship is that the animal naming and phonemic fluency tests require speech responses. Although the K-MBI includes domains thought to be important for measuring the activities of daily living [23], the lack of measures pertaining to communication, mood, and cognition can create an anomalous situation in which a dependent stroke survivor achieves a good MBI score [24]. Therefore, the K-MBI is primarily of value as a measure of physical dependency and is not applicable in studies that target speech disorders [25]. For this reason, there might be less of an association between the MBI score and fluency tests. Another potential explanation is that the digit symbol-coding test, TMT-A, and TMT-B are tests of psychomotor performance that are affected by motor speed and sustained attention [26].

Our study may have implications for which cognitive assessments should be recommended in clinical settings that are involved in stroke rehabilitation. Broad screening examinations, like the MMSE, are often preferred but have been criticized for being insensitive to varying impairments including visuospatial and executive functions. Our results support the use of screening measures that cover comprehensive functions in clinical settings. Furthermore, we suggest that more-complex neuropsychological test batteries like the K-VCIHS should be used in research examining long-term functional outcomes.

Several limitations of our study should be noted. First, we examined the predictive value of a neuropsychological battery in the 3 months immediately following a stroke with respect to the patient's functional assessment. Although it has been suggested that patients in the acute phase after a stroke are often unstable and difficult to test [27], our results show that an early neuropsychological examination is feasible in the majority of patients and has important prognostic value regarding their clinical assessment. Second, our study was not a population-based clinical trial (patients are usually re- 
ferred by general hospitals); thus, not all stroke survivors were enrolled. This selection bias may have resulted in an overestimation of the frequency of cognitive impairment. Therefore, our findings cannot be directly generalized to the entire population of patients who have experienced a stroke. However, since identifying the prognosis in the early stages following a stroke can be difficult, as is the adequate allocation of rehabilitation resources, our results may be useful in clinical practice because they may help quicken this process. Finally, our follow-up duration from stroke onset was restricted to 3 months. Future longitudinal studies should ideally follow patients who have experienced a stroke over a longer period of time.

In the present study, we found that VCI was highly prevalent in patients after a stroke. Here, among the tests of cognitive assessment employed, the TMT-A, which evaluates executive function, was highly predictive of patients' final K-MBI scores. Our findings suggest that post-stroke executive dysfunction, as measured by the K-VCIHS, is a significant and independent predictor of functional outcome. Therefore, the evaluation of executive function in stroke patients is recommended to predict functional outcome and to manage adequate rehabilitation. Based on our results, we suggest that more-complex neuropsychological test batteries be used to identify disabilities. Such efforts will hopefully improve the disability management and assessments in patients after a stroke.

\section{CONFLICT OF INTEREST}

No potential conflict of interest relevant to this article was reported.

\section{ACKNOWLEDGMENTS}

This study was supported by grants (No. HI10C2020, HI13C1990) from the Korean Health Technology R\&D Project and Ministry of Health \& Welfare (No. NRF2015R1C1A1A01055923, NRF-2017R1A2B4006500).

\section{REFERENCES}

1. Seshadri S, Wolf PA. Lifetime risk of stroke and dementia: current concepts, and estimates from the Framingham Study. Lancet Neurol 2007;6:1106-14.

2. Jin YP, Di Legge S, Ostbye T, Feightner JW, Hachinski
V. The reciprocal risks of stroke and cognitive impairment in an elderly population. Alzheimers Dement 2006;2:171-8.

3. Hachinski VC, Bowler JV. Vascular dementia. Neurology 1993;43:2159-60.

4. Gorelick PB, Scuteri A, Black SE, Decarli C, Greenberg SM, Iadecola $\mathrm{C}$, et al. Vascular contributions to cognitive impairment and dementia: a statement for healthcare professionals from the american heart association/american stroke association. Stroke 2011;42: 2672-713.

5. Delgado C, Donoso A, Orellana P, Vasquez C, Diaz V, Behrens MI. Frequency and determinants of poststroke cognitive impairment at three and twelve months in Chile. Dement Geriatr Cogn Disord 2010; 29:397-405.

6. Serrano S, Domingo J, Rodriguez-Garcia E, Castro MD, del Ser T. Frequency of cognitive impairment without dementia in patients with stroke: a two-year follow-up study. Stroke 2007;38:105-10.

7. Hachinski V, Iadecola C, Petersen RC, Breteler MM, Nyenhuis DL, Black SE, et al. National Institute of Neurological Disorders and Stroke-Canadian Stroke Network vascular cognitive impairment harmonization standards. Stroke 2006;37:2220-41.

8. Yu KH, Cho SJ, Oh MS, Jung S, Lee JH, Shin JH, et al. Cognitive impairment evaluated with Vascular Cognitive Impairment Harmonization Standards in a multicenter prospective stroke cohort in Korea. Stroke 2013;44:786-8.

9. Mann LL, Wong K. Development of an objective method for assessing viscosity of formulated foods and beverages for the dysphagic diet. J Am Diet Assoc 1996;96:585-8.

10. Joy S, Kaplan E, Fein D. Speed and memory in the WAIS-III Digit Symbol: coding subtest across the adult lifespan. Arch Clin Neuropsychol 2004;19:759-67.

11. Heruti RJ, Lusky A, Dankner R, Ring H, Dolgopiat M, Barell V, et al. Rehabilitation outcome of elderly patients after a first stroke: effect of cognitive status at admission on the functional outcome. Arch Phys Med Rehabil 2002;83:742-9.

12. O'Brien JT, Erkinjuntti T, Reisberg B, Roman G, Sawada T, Pantoni L, et al. Vascular cognitive impairment. Lancet Neurol 2003;2:89-98.

13. Nys GM, van Zandvoort MJ, de Kort PL, van der Worp 
HB, Jansen BP, Algra A, et al. The prognostic value of domain-specific cognitive abilities in acute first-ever stroke. Neurology 2005;64:821-7.

14. Wagle J, Farner L, Flekkoy K, Bruun Wyller T, Sandvik L, Fure B, et al. Early post-stroke cognition in stroke rehabilitation patients predicts functional outcome at 13 months. Dement Geriatr Cogn Disord 2011;31:37987.

15. Roman GC, Tatemichi TK, Erkinjuntti T, Cummings JL, Masdeu JC, Garcia JH, et al. Vascular dementia: diagnostic criteria for research studies. Report of the NINDS-AIREN International Workshop. Neurology 1993;43:250-60.

16. Park JH, Kim BJ, Bae HJ, Lee J, Lee J, Han MK, et al. Impact of post-stroke cognitive impairment with no dementia on health-related quality of life. J Stroke 2013;15:49-56.

17. Qu Y, Zhuo L, Li N, Hu Y, Chen W, Zhou Y, et al. Prevalence of post-stroke cognitive impairment in china: a community-based, cross-sectional study. PLoS One 2015;10:e0122864.

18. Petersen SE, Posner MI. The attention system of the human brain: 20 years after. Annu Rev Neurosci 2012; 35:73-89.

19. Liu T, Slotnick SD, Serences JT, Yantis S. Cortical mechanisms of feature-based attentional control.
Cereb Cortex 2003;13:1334-43.

20. Egner T, Monti JM, Trittschuh EH, Wieneke CA, Hirsch J, Mesulam MM. Neural integration of topdown spatial and feature-based information in visual search. J Neurosci 2008;28:6141-51.

21. Skidmore ER, Whyte EM, Holm MB, Becker JT, Butters MA, Dew MA, et al. Cognitive and affective predictors of rehabilitation participation after stroke. Arch Phys Med Rehabil 2010;91:203-7.

22. Kwakkel G, Wagenaar RC, Kollen BJ, Lankhorst GJ. Predicting disability in stroke: a critical review of the literature. Age Ageing 1996;25:479-89.

23. Donaldson SW, Wagner CC, Gresham GE. A unified ADL evaluation form. Arch Phys Med Rehabil 1973;54: 175-9.

24. Novak S, Johnson J, Greenwood R. Barthel revisited: making guidelines work. Clin Rehabil 1996;10:128-34.

25. Quinn TJ, Langhorne P, Stott DJ. Barthel index for stroke trials: development, properties, and application. Stroke 2011;42:1146-51.

26. Rossini ED, Karl MA. The Trail Making Test A and B: a technical note on structural nonequivalence. Percept Mot Skills 1994;78:625-6.

27. Laska AC, Hellblom A, Murray V, Kahan T, Von Arbin M. Aphasia in acute stroke and relation to outcome. J Intern Med 2001;249:413-22. 